\title{
Contralateral Suppression Of Teoae In Patients With Tinnitus and Normal Hearing
}

\author{
Kamala Sarathy* and V Jaya \\ Institute of speech and hearing, Madras Medical College, India
}

Received: October 25, 2017; Published: November 03, 2017

*Corresponding author: Kamala Sarathy, M.Sc Audiology, Faculty of Speech and Hearing, Institute of speech and hearing, Madras Medical College Chennai, India; Email:kamalasarathy.audio@gmail.com

\section{Abstract}

Aim and Objectives : To evaluate functional integrity of Outer Hair Cells and Medial Olivocochlear system in the subjects having normal hearing with tinnitus and compare it with normal population and also to study the importance of Otoacoustic Emissions testing as an important objective tool in tinnitus evaluation.

Methods and Materials: A total of 20 subjects in the age range of 18 years to 57 years were considered for the study. They were categorised into two groups such as experimental group and control group. Experimental group consisted of 14 subjects with normal hearing having unilateral or bilateral tinnitus. The control group consisted of 6 age matched subjects having normal hearing without any tinnitus. All the subjects of both the groups underwent Pure Tone Audiometry testing and tinnitus evaluation. TEOAE testing and Contralateral Suppression of TEOAE were carried out.

Results: Among the 19 ears in the experimental group, TEOAE was absent in 10 ears (52.6\%) and present in 9 ears (47.3\%). Presence of contralateral suppression of TEOAE was observed in 6 ears (31.5\%) and absence of contralateral suppression was noted in 13 ears (68.4\%). It can be observed that most of patients in the experimental group had absent TEOAE and there is a subtle difference in the contralateral suppression of TEOAE between the experimental and control group.

Conclusion: In conclusion abnormal OAE in patients with tinnitus having normal hearing sensitivity indicate the cochlear dysfunction. Absence of suppression indicates Medial Olivocochlear system dysfunction. We also suggest that other auditory structures and mechanisms apart from OHC and MOC system may also be the reason for tinnitus generation as the results included patients with normal OAE and Contralateral suppression of OAE.The study helps to understand the role of OAE measures in evaluating the functional integrity of Outer Hair Cells and MOC system in subjects having tinnitus with normal hearing. It also highlight about the role of MOC system and cochlea in the generation of tinnitus.

Keywords: Tinnitus; CSOAE; TEOAE; MOC system; Outer Hair Cells

Abbreviations: OAEs: Otoacoustic Emissions; MOC: Medial Olivocochlear System; MNTB: Medial Nucleus of the Trapezoid Body; OHCs: Outer Hair Cells; TEOAE: Transient Evoked Otoacoustic Emissions

\section{Introduction}

Tinnitus represents one of the most common and distressing otologic problems which cause various somatic and psychological disorders that interfere with the quality of life [1]. Perception of sound in the ears or head that lacks an external acoustic source is commonly defined as tinnitus or ringing in the ears [2]. The majority of tinnitus patients have hearing loss but it can also occur in patients with normal hearing [3]. Several theories have been proposed for origin of tinnitus inspite of that the exact mechanism behind the generation of tinnitus is poorly understood. Tinnitus may be associated with abnormalities in any level of the auditory pathways. Jastreboff considers that the tinnitus is due to the maladaptive plasticity changes includes the enhanced central gain due to the compensatory increase in the central auditory activity in response to the loss of sensory input and the abnormal emotional reactions associated with the tinnitus. Several studies have investigated the relationship between tinnitus and dysfunction of the efferent auditory system mainly the Medial Olivocochlear System (MOC) by the suppression of otoacoustic emissions (OAEs) .

The Medial Olivocochlear System (MOC) is one of the efferent auditory system. The Medial Olivocochlear bundle arises from the neurons of the Medial Superior Olivary (MSO) nucleus complex and the Medial Nucleus of the Trapezoid Body (MNTB) and comprises of thick myelinated nerve fibres. About $75 \%$ of the fibers cross at the floor of fourth ventricle and terminate to the outer Hair Cells (OHCs) of the contralateral cochlea, while the rest of them remain uncrossed and terminate to the Outer Hair Cells (OHCs) 
of the ipsilateral cochlea. The fibers of the Olivocochlear bundle synapse directly at the basal surface of the Outer Hair Cells. The role of the efferent auditory system remains largely unknown. In view of preferential innervation of the OHCs by MOC system, it has been hypothesized that stimulation of Medial efferent alters IHC sensitivity indirectly by altering the micromechanical properties of the the OHCs. It is well established that length, tension and stiffness of the OHCs along their longitudinal axis are under the control of MOC bundle, thus enhancing the auditory sensitivity for low level stimuli at 30 to $40 \mathrm{~dB}$ SL.

The Medial Olivocochlear bundle is mainly inhibitory. Hence there has been already suggestions that dysfunction of the efferent auditory system at any level auditory cortex to cochlea may be a basis for tinnitus generation [4]. The contralateral suppression of Otoacoustic Emissions (OAEs) could serve as an objective and non invasive clinical tool for exploration of the non-linear micromechanical of OHCs and clinical neurologic evaluation of the auditory brainstem especially the MOC system. The contralateral suppression of OAEs is performed by measuring OAE from the test ear while the contralateral ear is stimulated with noise. The difference in the OAE amplitude with and without contralateral noise stimulation is calculated . Negative value or zero indicate no suppression while positive values indicate suppression of OAEs . A cut off of $0.5 \mathrm{~dB}$ SPL is considered as suppression. The present study is aimed to evaluate functional integrity of $\mathrm{OHC}$ and MOC system in the subjects having normal hearing with tinnitus and compare it with normal population and also to study the importance of OAE testing as an important objective tool in tinnitus evaluation.

\section{Methods and Materials}

A total of 28 subjects in the age range of 18 years to 57 years were considered for the study. They were categorised into two groups such as experimental group and control group. Experimental group consisted of 14 subjects ( 5 female and 9 male) with normal hearing having unilateral or bilateral tinnitus. 9 subjects had unilateral tinnitus and 5 subjects had bilateral tinnitus. A total of 19 ears were considered for the study. The control group consisted of 14 (28 ears) age matched subjects having normal hearing without any tinnitus. All the subjects of both the groups underwent Pure Tone Audiometry testing. Pure Tone thresholds within $25 \mathrm{dBHL}$ in all octave frequencies from $250 \mathrm{~Hz}$ to $8000 \mathrm{~Hz}$ was considered as normal hearing sensitivity. Inventis Piano audiometer with TDH 39 supra aural headphone and BC71 bone vibrator was used for testing. After the Pure Tone Testing, tinnitus evaluations were performed over all the subjects. Tinnitus evaluations included pitch and loudness matching test. Pure tones and Narrow Band Noises were used according to the range of loudness and frequency.

11 kinds of frequencies were used for pitch matching (125 $\mathrm{Hz}, 250 \mathrm{~Hz}, 500 \mathrm{~Hz}, 750 \mathrm{~Hz}, 1000 \mathrm{~Hz}, 1500 \mathrm{~Hz}, 2000 \mathrm{~Hz}, 3000 \mathrm{~Hz}$, $4000 \mathrm{~Hz}, 6000 \mathrm{~Hz}, 8000 \mathrm{~Hz}$ ). The patients were instructed to match the pitch of the external tone to the pitch of the tinnitus. The tone was presented in the ear contralateral to the tinnitus ear to avoid residual inhibition. Three trials of pitch matching with an interval of 1 minute were given. Once the pitch of tinnitus was established, the patients were instructed to match the loudness of the tinnitus to external tone presented in the contralateral ear to the tinnitus. IHS system was used for Transient Evoked Otoacoustic Emissions (TEOAE) testing and contralateral suppression of TEOAE. TEOAE measurements were recorded by presenting clicks of duration of 40 microseconds. 1024 sweeps of $80 \mathrm{~dB}$ peak SPL were presented and TEOAE amplitude at each frequency band of $1 \mathrm{kHz}, 1.5 \mathrm{kHz}$, $2 \mathrm{kHz}, 3 \mathrm{kHz}$ and $4 \mathrm{kHz}$ were considered as baseline for contralateral suppression of TEOAE. Contralateral suppression of TEOAE testing included recording of amplitude, SNR and reproducibility in the presence of continuous white noise presented at $50 \mathrm{~dB}$ SPL through insert earphones. Difference in baseline TEOAE amplitude and TEOAE amplitude measured in the presence of contralateral noise is calculated at each octave frequencies of $1 \mathrm{kHz}, 1.5 \mathrm{kHz}, 2 \mathrm{kHz}$, $3 \mathrm{kHz}$ and $4 \mathrm{kHz}$. Positive values indicate presence of suppression and negative value or zero indicate absence of suppression. $0.5 \mathrm{~dB}$ SPL was considered as the presence of suppression.

\section{Results}

Table 1: Number and Percentage of ears that had absent TEOAE across different frequencies in the experimental group.

\begin{tabular}{|c|c|c|}
\hline Frequency & Number Of Ears & Percentage \\
\hline $1 \mathrm{kHz}$ & 12 & $63.1 \%$ \\
\hline $1.5 \mathrm{kHz}$ & 9 & $47.3 \%$ \\
\hline $2 \mathrm{kHz}$ & 11 & $57.8 \%$ \\
\hline $3 \mathrm{kHz}$ & 7 & $36.8 \%$ \\
\hline $4 \mathrm{kHz}$ & 11 & $57.8 \%$ \\
\hline
\end{tabular}

Table 2: Number and Percentage of ears that had absence of contralateral suppression of TEOAE across different frequencies in the experimental group.

\begin{tabular}{|c|c|c|}
\hline Frequency & Number Of Ears & Percentage \\
\hline $1 \mathrm{kHz}$ & 0 & $0 \%$ \\
\hline $1.5 \mathrm{kHz}$ & 8 & $42.1 \%$ \\
\hline $2 \mathrm{kHz}$ & 9 & $47.3 \%$ \\
\hline $3 \mathrm{kHz}$ & 13 & $68.4 \%$ \\
\hline $4 \mathrm{kHz}$ & 10 & $52.6 \%$ \\
\hline
\end{tabular}

Table 3: Number and Percentage of ears that had absence of contralateral suppression of TEOAE across different frequencies in the control group.

\begin{tabular}{|c|c|c|}
\hline Frequency & Number Of Ears & Percentage \\
\hline $1 \mathrm{kHz}$ & 4 & $14.2 \%$ \\
\hline $1.5 \mathrm{kHz}$ & 4 & $14.2 \%$ \\
\hline $2 \mathrm{kHz}$ & 5 & $17.8 \%$ \\
\hline $3 \mathrm{kHz}$ & 9 & $32.1 \%$ \\
\hline $4 \mathrm{kHz}$ & 8 & $28.5 \%$ \\
\hline
\end{tabular}

Among the 19 ears in the experimental group, TEOAE was absent in 10 ears (52.6\%) and present in 9 ears (47.3\%). Presence of contralateral suppression of TEOAE was observed in 6 ears (31.5\%) and absence of contralateral suppression was noted in 13 ears (68.4\%) From (Table 1), it can be observed that most of patients in the experimental group had absent TEOAE. From 
(Tables $2 \& 3$ ), it can be observed that there is a subtle difference in the contralateral suppression of TEOAE between the experimental and control group.

\section{Discussion}

The results obtained from the present study are comparable to other studies. A study done by Ceranic et al in 1995 shown that OAE are not normal at tinnitus frequency region even in subjects with normal hearing [5]. Another study done by Almeida et al in 2006 indicated that TEOAEs were abnormal in $70.2 \%$ of individuals with tinnitus having normal hearing [6]. Study done by Paglialonga et al in 2010 revealed that $13 \%$ of patients with tinnitus exhibited abnormal TEOAE [7]. One of the study done by Dhanya et al in 2009 reported absent TEOAE in $47.3 \%$ of individuals with tinnitus having normal hearing sensitivity [8]. Study done by Serra reported absent TEOAE in $67 \%$ of individuals with normal hearing and tinnitus [9] in a study pointed out slightly reduced TEOAE suppression in tinnitus subjects compared to non- tinnitus ears. Ryan and Kemp found a large range of suppression variability in the contralateral suppression of OAE [10]. Study by Dhanya et al. [9] also revealed a high variability in contralateral suppression of OAE. A study by Geven found that there was no significant difference in the amount of suppression between tinnitus patients and control group. Similar findings were obtained in our study $[11,12]$.

\section{Conclusion}

We conclude from the present study that abnormal OAE in patients with tinnitus having normal hearing sensitivity indicate the cochlear dysfunction. Absence of suppression indicates Medial Olivocochlear system dysfunction. We also suggest that other auditory structures and mechanisms apart from $\mathrm{OHC}$ and MOC system may also be the reason for tinnitus generation as the results included patients with normal OAE and Contralateral suppression of OAE. The study helps to understand the role of OAE measures in evaluating the functional integrity of Outer Hair Cells and MOC system in subjects having tinnitus with normal hearing.
It also highlight about the role of MOC system and cochlea in the generation of tinnitus.

\section{References}

1. Yetsier S, Tosun F, Satar B, Arslanhan M, Akcam T, et al. (2002) The Role of Zinc In Management Of Tinnitus. Auris Nasus Larynx 29(4): 329-333.

2. Jastreboff PJ (1990) Phantom Auditory Perception(Tinnitus): Mechanisms of Generation and Perception. Neuro sci Res 8(4): 221-254.

3. Sanchez TG, Mak MP, Pedalini MEB, Levy CPD, Bento RF (2005) Tinnitus and Hearing Evolution in Normal Hearing Patients. Archives of Otolaryngology 9(3): 227-330.

4. Prasher D, Ceranic B, Sulkowski W, Guzek W (2001) Objective evidence for tinnitus from spontaneous emission variability. Noise Health 3(12): 61-73.

5. In Henderson D, Prasher D, Kopke R, Salvi R, Hamernik R (2001) Noise Induced Hearing Loss: Basic Mechanisms Prevention and Control. Noise Research Publications London 471-483.

6. Ceranic BJ, Prasher DK, Luxon LM (1995) Tinnitus and Otoacoustic Emissions. Clinical Otolaryngology 20(2): 192-200.

7. Almeida VF, Granjeiro RC, Sampaio ALL, Furtado V (2006) Otoacoustic Emissions in Patients with Tinnitus. Otolaryngology- Head and Neck Surgery 135(2): 245-246.

8. Pagliolonga A, Del Bo L, Ravazzania P, Tognolaa G (2010) Quantitave analysis of cochlear active mechanisms in tinnitus subjects with normal hearing sensitivity: multiparametric recording of evoked otoacoustic emissions and contralateral suppression 37(3): 291-298.

9. Dhanya M, Barman A (2009) OAE profile in Individuals with Tinnitus having Normal Hearing Sensitivity.

10. Luciency Serra, Gabriela Novanta, Andre Lopes Sampaio, Carlos Augusto Oliveira, Ronaldo Granjeiro, et al. (2015) Int Arch Otorhinolaryngol 19(2): 171-175.

11. Ryan S, Kemp DT (1996) The Influence of evoking stimulus level on the neutral suppression of transient evoked otoacoustic emissions. Hearing Research 84(2): 140-147.

12. Leontien I Geven, Emile de Kleine, Rolien H Free, Pim van Dijk (2011) Contralateral Suppression of Otoacoustic Emissions in Tinnitus Patients. Otology \& Neurology 32(2): 315-321.

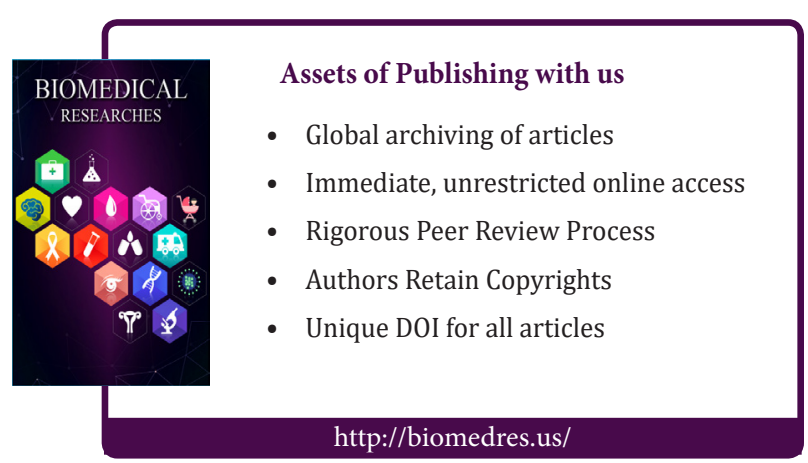

